TRANSACTIONS OF THE

AMERICAN MATHEMATICAL SOCIETY

Volume 362, Number 8, August 2010, Pages 4213-4228

S 0002-9947(10)04871-3

Article electronically published on March 23, 2010

\title{
A RELATIVE OKA-GRAUERT PRINCIPLE FOR HOLOMORPHIC SUBMERSIONS OVER 1-CONVEX SPACES
}

\author{
JASNA PREZELJ
}

\begin{abstract}
This paper presents the proof of the relative Oka-Grauert principle for holomorphic submersions over 1-convex spaces using conic neighbourhoods of holomorphic sections over 1-convex spaces. A proof of a version of Cartan's Theorem $A$ for 1-convex spaces is also given.
\end{abstract}

\section{INTRODUCTION AND THE MAIN THEOREM}

The Oka-Grauert principle says that on Stein spaces cohomologically formulated analytic problems have only continuous obstructions. In particular, if there exists a continuous solution of such a problem, then there is also an analytic one, and the two can be joined by a homotopy. Oka was the first to get the results of this type for solutions of Cousin problems. A solution of the second Cousin problem on a Stein manifold $X$ can be viewed as a (global) holomorphic section of a holomorphic $\mathbb{C}^{*}$ bundle over $X$. This was generalized by Grauert to sections of holomorphic bundles with complex homogeneous fibres. Many other generalizations followed (Gromov Gro, Forstnerič [F1], F2], Forstnerič-Prezelj [FP1], Forstnerič-Slapar [FS]), but in all these cases the base space was still Stein.

The relative Oka-Grauert principle for a holomorphic vector bundle over a 1convex manifold was proved by Henkin and Leiterer ([HL]). Recently, Leiterer and Vâjâitu ([LV]) proved the validity of the relative Oka-Grauert principle for a holomorphic bundle over a 1-convex space, the characteristic fibre of which is a complex Lie group.

Recall that a complex space $X$ is 1-convex if it possesses a plurisubharmonic exhaustion function which is strictly plurisubharmonic outside a compact set. It is known that the Remmert reduction of a 1-convex space $X$ is Stein. Therefore the following conclusion can be expected: if $h: Z \rightarrow X$ is a holomorphic submersion from a complex space to a 1-convex space and $a: X \rightarrow Z$ is a continuous section of the submersion which is already holomorphic on a neighbourhood of the exceptional set, then $a$ is homotopic to a holomorphic one. This is a formulation of the relative Oka-Grauert principle for sections of holomorphic submersions (compare [LV]). For more details on 1-convex spaces we refer the reader to the following papers: An-

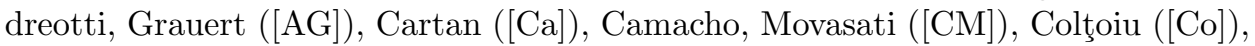

Received by the editors August 21, 2007 and, in revised form, June 1, 2008.

2010 Mathematics Subject Classification. Primary 32C15, 32C25, 32L05, 32L10, 32E05, 32E10, 32E30, 32C35.

Key words and phrases. Stein space, 1-convex space, relative Oka-Grauert principle, conic neighbourhoods.

The author was supported by research program P1 - 0291, Slovenia.

(C) 2010 American Mathematical Society Reverts to public domain 28 years from publication 
Demailly ([De] $)$, Grauert ([G1], G2]), Leiterer-Vâjâitu ([LV]), Siu ([S1]), Vâjâitu ( $\mathrm{Va}]$ ).

The main result of the present paper is the following:

Theorem 1.1 (The relative Oka-Grauert principle for 1-convex spaces). Let $X$ be a reduced 1-convex space, $S$ its exceptional set, $Z$ a complex space and $h: Z \rightarrow X$ a holomorphic submersion having the Runge property over the set $X \backslash S$ (see the definition below).

Let $K \subset X$ be a holomorphically convex compact subset of $X$ containing $S$ and $a_{0}: X \rightarrow Z$ a continuous section of the submersion, holomorphic on a neighbourhood of $K$. Then there exists a homotopy $a_{t}: X \rightarrow Z$ such that

(1) $a_{t}(x)=a_{0}(x)$ for $x \in S$,

(2) the sections $a_{t}$ are holomorphic on a neighbourhood of $K$ and approximate $a_{0}$ on $K$ as well as desired,

(3) $a_{1}$ is holomorphic on $X$.

Remark 1.2. The set $S$ may be empty. Then the theorem provides a proof for the $h$-principle for submersions with sprays over Stein spaces.

Theorem 1.3 (Generalizations of the main theorem). Let $X, S, K$ be as in Theorem 1.1, $h: Z \rightarrow X$ a holomorphic submersion with a locally dominating spray over $(X \backslash S), P$ a compact, $T_{2}$ set of parameters and $g_{1}, \ldots, g_{m}: X \rightarrow \mathbb{C}$ holomorphic functions satisfying $\bigcap g_{i}^{-1}(0)=S$. Define $G=\bigcup g_{i}^{-1}(0)$ and let $a_{0, p}: X \rightarrow Z$ be a continuous family of continuous sections, holomorphic on a neighbourhood $U$ of $K$ in $X$. The Theorem 1.1 can be generalized to the following cases:

(a) Parametric version: if the family $a_{0, p}: X \rightarrow Z$ of continuous sections, which is continuous (smooth) in parameter $p$, satisfies $a_{0, p}(x)=a_{0, q}(x)$ for $p, q \in P, x \in G$, we get a homotopy $a_{t, p}: X \rightarrow Z$ such that $a_{t, p}(x)=a_{0, p}(x)$ for $x \in G$, the sections $a_{t, p}$ are holomorphic on a neighbourhood of $K$, the section $a_{1, p}$ is holomorphic on $X$ and homotopy is continuous (smooth) in $p$.

(b) Keeping some sections fixed: if in addition to (a) there is a subset $P_{0} \subset P$ of parameters, such that $P_{0}$ is a strong deformation retract of some neighbourhood $P_{1} \subset P$ of $P_{0}$ and $a_{0, p}: X \rightarrow Z, p \in P_{0}$ already is holomorphic, then $a_{t, p}=a_{0, p}, p \in P_{0}$.

(c) Interpolation: if the initial section is holomorphic on an analytic subset $X^{\prime} \subset X$, then the homotopy can be fixed on $X^{\prime}$.

Remark 1.4. The set $P_{0}$ is called a nice compact set (see definition 1.4 in [FP2]).

Remark 1.5. The condition that the initial sections must coincide on $G$ is only needed for Lemma 4.1. It may not be necessary.

Remark 1.6. Assume that $S$ is empty. Then we may take for example $g_{i}=1$ and therefore impose no conditions on the initial sections. In this case Theorem 1.3 says that every holomorphic section of $Z$ defined over Stein space $X^{\prime} \subset X$ of an arbitrary complex space $X$ has a holomorphic extension to a thin (Stein) neighbourhood of $X^{\prime}$ in $X$ since there is always a continuous extension to some neighbourhood of $X^{\prime}$ in $X$.

Definition 1.7 (The Runge property). Let $X$ be an arbitrary complex space, $S \subset X$ a subset and $h: Z \rightarrow X$ a holomorphic submersion. A holomorphic 
submersion $h: Z \rightarrow X$ has the Runge property (or fulfills the Runge condition) over $X \backslash S$ if every point $x \in X \backslash S$ has an open (Stein) neighbourhood $U_{x}$ such that the following holds. Let $P_{0}, P_{1}$ and $P$ be as in Theorem 1.3 (b), and let $C \subset U_{x}$ be a holomorphically convex compact set and continuous family of continuous sections $c_{p, t}: U_{x} \rightarrow Z, p \in P, t \in[0,1]$ with the following properties:

(1) $c_{p, t}$ is holomorphic on a neighbourhood of $C$ for every $p \in P, t \in[0,1]$,

(2) the sections $c_{p, 0}, p \in P$ and $c_{p, t}, p \in P_{1}$ and $t \in[0,1]$ are holomorphic on $U_{x}$.

Then there exists a homotopy of continuous families $c_{,,-}^{s}: U_{x} \rightarrow Z, s \in[0,1]$ such that

(1) $c_{p, t}^{0}=c_{p, t}$ for all $p \in P, t \in[0,1]$,

(2) for each $t \in[0,1], p \in P$, the sections $c_{p, t}^{s}, s \in[0,1]$ approximate the section $c_{p, t}$ on $C$ as well as desired,

(3) $c_{p, t}^{s}=c_{p, t}$ for $t, s \in[0,1], p \in P_{0}$.

Only the following examples of submersions fulfilling the Runge condition are known to the author:

Proposition 1.8. Let $X, S, Z$ be as above. The submersion $h: Z \rightarrow X$ fulfills the Runge condition over $X \backslash S$ in the following cases:

(a) $X$ is a manifold and $h: Z \rightarrow X$ is a bundle with fibres having the CAP property ([F1]),

(b) $X$ is a manifold and $h: Z \rightarrow X$ is a submersion with a locally dominating spray over $X \backslash S$ (Gro, [FP2]),

(c) $X$ is a reduced Stein space and $h: Z \rightarrow X$ a stratified submersion (F. Forstnerič, work in preparation).

One of the main tools in proving the Oka-Grauert principle for Stein spaces is the existence of Stein neighbourhoods of embedded Stein spaces ([De, S2]). This cannot be generalized to the 1-convex case in the sense that there is a 1convex neighbourhood of embedded 1-convex space. Since it is known that by removing an appropriate analytic set from $X$ we get a Stein space, one would try to use this fact for construction of Stein neighbourhoods of a certain shape. The main technical contribution of the paper is the theorem on the existence of conic Stein neighbourhoods (see the definition in section 2) of Stein sets of the form $a\left(U \backslash g^{-1}(0)\right)$, where $U$ is 1-convex, $a: U \rightarrow Z$ a holomorphic section and $g: X \rightarrow \mathbb{C}$ a holomorphic function such that $g(S)=0$ (keep in mind that $S$ denotes the exceptional set). The term conic refers to the shape of the neighbourhood of $a\left(U \backslash g^{-1}(0)\right)$ when approaching $a\left(g^{-1}(0)\right)$ : the width of the neighbourhood (in the vertical direction) should not decrease faster than polynomially, i.e. it should lie between two cones. The existence of such neighbourhoods enables us to make small perturbations of the section $a$, fixing $S$ as images of small sections of a trivial bundle over $a(U)$ obtained by integrating holomorphic vector fields with zeroes on $S$. If the order of the zeroes is high enough, then there exists a small $t_{0}$ such that the flows of the vector fields do not escape from the conic neighbourhood for times smaller than $t_{0}$ (details are in section 3 ). It should be mentioned that the Demailly and Siu result gives a Stein neighbourhood of the set $a\left(U \backslash g^{-1}(0)\right)$, but in general the width of it decreases exponentially when approaching $g^{-1}(0)$.

The structure of the paper is the following: in the second section some tools such as the Runge theorems and Cartan's Theorems A and B are presented for 
1-convex spaces. The third section consists of the theorem on existence of conic neighbourhoods and the fourth section contains the proof of the main theorem.

\section{TOOLS}

From now on we assume that $\left(X, \mathcal{O}_{X}\right)$ is a reduced 1-convex space, $S$ its exceptional set and $\pi: X \rightarrow Y$ the Remmert reduction.

Proposition 2.1 (Lemma 3.3 in [LV]). Let $V \Subset Y$ be a Stein open set and $U:=$ $\pi^{-1}(V)$. Then for any coherent analytic sheaf $\mathcal{Q}$ on $X$ the following holds:

(1) There are finitely many sections in $\Gamma(X, \mathcal{Q})$, the restrictions of which generate $\Gamma(U, \mathcal{Q})$ as a $\Gamma(U, \mathcal{O})$ module.

(2) If $V$ is holomorphically convex in $Y$, then $\Gamma(X, \mathcal{Q}) \rightarrow \Gamma(U, \mathcal{Q})$ has a dense image.

Remark 2.2. Let $K \subset U$ be a compact set of the form $K=\pi^{-1}(\pi(K))$. The second assertion in the case of vector bundles says that if $V \rightarrow X$ is a holomorphic vector bundle, then any vector field over $U$ can be approximated on $K$ by a global one.

Lemma 2.3. Let $M \subset Y$ be a holomorphically convex set and $K=\pi^{-1}(M)$. Then $K$ has a basis of Runge neighbourhoods in $X$.

Proof. According to the classical theory the set $M$ has a basis of holomorphically convex neighbourhoods in $Y$. The rest follows from Proposition 2.1.

Theorem 2.4 (Theorem B for 1-convex spaces, G1, Satz 2, p. 357]). Let $\mathcal{Q}$ be a coherent analytic sheaf on $X, \mathcal{J}=\mathcal{J}(S)$, the ideal generated by $S$ and let $U \Subset X$ be a strictly pseudoconvex set containing $S$. There exists a positive integer $n_{0}$ such that $H^{k}\left(U, \mathcal{Q J}^{n}\right)=0$ for all $k \in \mathbb{N}$ and $n \geq n_{0}$.

In the case of Stein spaces Theorem A is a consequence of Theorem B as proved in Theorem 2, p. 101 in GR1. Following the proof of Theorem 2, we can also get a following version of Theorem A:

Theorem 2.5 (Theorem A for 1-convex spaces). Let $X$ be a 1-convex space with an exceptional set $S, U \Subset X$ a strictly pseudoconvex set containing $S, \mathcal{J}=\mathcal{J}(S)$ the ideal sheaf generated by the set $S$ and $\mathcal{Q}$ a coherent sheaf on $X$. There exists an $n_{0} \in \mathbb{N}$ such that for $n \geq n_{0}$ the sheaf $\mathcal{Q} \mathcal{J}^{n}$ is locally generated by $\Gamma\left(U, \mathcal{Q} \mathcal{J}^{n}\right)$ on $U$.

Proof. Choose an arbitrary strictly pseudoconvex set $U$ such that $V \Subset U$. We divide the proof into three steps. For each $x_{0} \in X$ let $\mathcal{N}\left(x_{0}\right)$ be a (coherent) sheaf defined by $\mathcal{N}\left(x_{0}\right)_{x}=\mathcal{O}_{X, x}$ for $x \neq x_{0}$ and $\mathcal{N}\left(x_{0}\right)_{x_{0}}=m_{x_{0}}$, where $m_{x_{0}}$ denotes the maximal ideal in $\mathcal{O}_{X, x_{0}}$.

(1) There exists an $n_{x_{0}}$ such that for $n \geq n_{x_{0}}$ there are finitely many global sections of $\mathcal{Q} \mathcal{J}^{n}$ that generate the sheaf $\mathcal{Q} \mathcal{J}^{n}$ at $x_{0}$.

Proof. The short exact sequence

$$
0 \rightarrow \mathcal{N}\left(x_{0}\right) \mathcal{Q} \mathcal{J}^{n} \rightarrow \mathcal{Q} \mathcal{J}^{n} \rightarrow \mathcal{Q} \mathcal{J}^{n} /\left(\mathcal{N}\left(x_{0}\right) \mathcal{Q} \mathcal{J}^{n}\right) \rightarrow 0
$$

generates the long exact sequence

$$
\ldots \rightarrow \Gamma\left(U, \mathcal{Q J}^{n}\right) \rightarrow \Gamma\left(U, \mathcal{Q} \mathcal{J}^{n} /\left(\mathcal{N}\left(x_{0}\right) \mathcal{Q} \mathcal{J}^{n}\right)\right) \rightarrow H^{1}\left(U, \mathcal{N}\left(x_{0}\right) \mathcal{Q} \mathcal{J}^{n}\right) \rightarrow \ldots
$$


By Theorem 2.4 there exists $n_{x_{0}} \in \mathbb{N}$ such that $H^{1}\left(U, \mathcal{N}\left(x_{0}\right) \mathcal{Q} \mathcal{J}^{n}\right)=0$ for $n \geq n_{x_{0}}$. For such $n$ the induced map

$$
d: \Gamma\left(U, \mathcal{Q} \mathcal{J}^{n}\right) \rightarrow \Gamma\left(U, \mathcal{Q} \mathcal{J}^{n} / \mathcal{N}\left(x_{0}\right) \mathcal{Q} \mathcal{J}^{n}\right)
$$

is surjective. The quotient $\mathcal{Q J}^{n} / \mathcal{N}\left(x_{0}\right) \mathcal{Q J}^{n}$ is 0 for $x \neq x_{0}$ and a finite dimensional vector space for $x=x_{0}$. By Nakayama's lemma (GR1], p. 101) there are finitely many sections from $\Gamma\left(U, \mathcal{Q J}^{n}\right)$ that generate the sheaf $\mathcal{Q J}^{n}$ at $x_{0}$.

(2) The proposition holds for $\mathcal{Q}=\mathcal{J}$.

Since the Remmert reduction $\pi(X)=Y$ is Stein, then for each $n \in \mathbb{N}$ there exist functions $g_{1, n}, \ldots, g_{m_{n}, n} \in \Gamma\left(Y, \mathcal{J}^{n}(\pi(S))\right)$ with the set $\pi(S)$ as their common zero set. Then the functions $g_{j} \circ \pi \in \Gamma\left(X, \mathcal{J}^{n}\right)$ have $S$ as their common zero set and generate $\mathcal{J}^{n}$ on $X \backslash S$.

Now we have to produce functions defined on $U$ that would generate the sheaves $\mathcal{J}^{n}$ on $S$ for all large $n$ 's. Take an arbitrary $x \in S$ and let $n_{x} \in \mathbb{N}$ be such that $H^{1}\left(U, \mathcal{N}(x) \mathcal{J}^{n}\right)=0$ for $n \geq n_{x}$. Fix an $n \geq n_{x}$. This means that there exist finitely many holomorphic functions $f_{1}, \ldots, f_{k} \in \Gamma\left(U, \mathcal{J}^{n}\right)$ which generate $\mathcal{J}_{x}^{n}$.

By the argument above, we may assume $N\left(f_{1}, \ldots, f_{k}\right) \subset S$. The functions $f_{1}, \ldots, f_{m}$ define a sheaf homomorphism

$$
F=\left(f_{1}, \ldots, f_{k}\right): \mathcal{O}^{m} \rightarrow \mathcal{J}^{n} .
$$

Then the sheaf Coker $(F)$ is coherent, and its support consists of points where the map $F_{x}: \mathcal{O}_{x}^{m} \rightarrow \mathcal{J}_{x}^{n}$ is not surjective, i.e. $\mathcal{J}_{x}^{n}$ is not generated by sections $f_{1}, \ldots, f_{k}$. The set $\operatorname{supp}(\operatorname{Coker}(F))$ is then an analytic subset of a compact analytic set $S$ and therefore has only finitely many irreducible components $S_{1}, \ldots, S_{l}$. Let $s_{i} \in \bigcap_{i \neq j} S_{i} \backslash S_{j}$. By Theorem B we have $H^{1}\left(U, \mathcal{N}\left(s_{j}\right) \mathcal{J}^{N}\right)=0$ for all sufficiently large $N$. Take such a large $N$ of the form $N=j n$ and let $f_{k+1}, \ldots, f_{m} \in \Gamma\left(U, \mathcal{J}^{N}\right)$ be global sections generating $\mathcal{J}^{N}$ at $s_{1}, \ldots, s_{l}$. Let $\alpha=\left(\alpha_{1}, \ldots, \alpha_{j}\right) \in\{1, \ldots, k\}^{j}$ be a $j$-tuple of integers and

$$
f_{\alpha}=\prod_{i=1}^{j} f_{\alpha_{i}} .
$$

Then the functions $f_{\alpha}$ generate the sheaf $\mathcal{J}^{N}$ off $\operatorname{supp}(\operatorname{Coker}(F))$. The set where the functions $f_{\alpha}$, together with $f_{k+1}, \ldots, f_{m}$, do not generate the sheaf $\mathcal{J}^{N}$ is again an analytic subset of $\operatorname{supp}(\operatorname{Coker}(F))$ with dimension smaller than $\operatorname{dim} \operatorname{supp}(\operatorname{Coker}(F))$. The rest follows by induction.

We have produced a finite number of (global) holomorphic functions generating $\mathcal{J}^{N}$ on $U$ for some large $N>n_{x}$. Their mutual products then generate the sheaves $\mathcal{J}^{2 N}, \mathcal{J}^{3 N}, \mathcal{J}^{4 N}$ etc. on $U$. Let $F_{1}^{m}, \ldots, F_{l_{m}}^{m} \in \Gamma\left(U, \mathcal{J}^{m N}\right)$ denote the holomorphic functions generating $\mathcal{J}^{m N}$ on $U$.

By (1) there exists $j_{x} \in \mathbb{N}$ and an open neighbourhood $U_{x}$ of $x$ such that for each $n=j_{x} N, \ldots,\left(j_{x}+1\right) N-1$ there are finitely many functions $f_{1}^{n}, \ldots, f_{k_{n}}^{n} \in \Gamma\left(U, \mathcal{J}^{n}\right)$ generating $\mathcal{J}^{n}$ on $U_{x}$. The products $f_{i}^{n} F_{j}^{m}$ generate $\mathcal{J}^{n+m N}$ on $U_{x}$ for each $m \in \mathbb{N}$ and each $n=j N, \ldots,(j+1) N-1$, i.e. all the sheaves $\mathcal{J}^{k}, k \geq j N$, are generated on $U_{x}$ by finitely many global sections over $U$. 
The set $S$ has a finite open covering with the sets $U_{x_{1}}, \ldots, U_{x_{l}}$ as above. Let $m_{S}:=\max \left\{j_{x_{i}} N, i=1, \ldots, l\right\}$. Then the sheaves $\mathcal{J}^{k}, k \geq m_{S}$, are generated on $\bigcup_{i} U_{x_{i}} \supset S$ by finitely many global sections of $\mathcal{J}^{k}$ over $U$. Adding the functions $g_{1, k} \circ \pi, \ldots, g_{m_{k}, k} \circ \pi \in \Gamma\left(X, \mathcal{J}^{k}\right)$, we obtain (for each $\left.k \geq m_{S}\right)$ a finite number of functions defined over $U$ generating $\mathcal{J}^{k}$ on $U$.

(3) The proposition holds for an arbitrary coherent sheaf $\mathcal{Q}$.

By the direct image theorem (or Proposition 2.1) any coherent sheaf on $X$ is locally generated by finitely many global sections off the exceptional set. Take $x \in U$. Let $n_{x} \in \mathbb{N}$ be such that $H^{1}\left(U, \mathcal{N}(x) \mathcal{Q} J^{n}\right)=0$ for $n \geq n_{x}$. Fix such an $n$. There exist finitely many sections $g_{1}, \ldots, g_{l}$ generating $\mathcal{Q} J^{n}$ on some open neighbourhood $U_{x}$ of $x$. According to (2) there exists an $m_{S} \in \mathbb{N}$ such that for each $m \geq m_{S}$ there exist finitely many functions $f_{1}^{m}, \ldots, f_{k_{m}}^{m}$ generating $\mathcal{J}^{m}$ on $U$. Then the sections $g_{i} f_{j}^{m}, i=1, \ldots, l, j=1, \ldots, k_{m}$ generate $\mathcal{Q} J^{n+m}$ on $U_{x}$ for each $m \geq m_{S}$. By compactness of $S$ we conclude that the proposition holds for all coherent sheaves.

Remark 2.6. Notation as in (3) above. Note that by Nakayama's lemma the map

$$
\Gamma\left(U, \mathcal{Q} \mathcal{J}^{n}\right) \rightarrow \Gamma\left(U,\left(\mathcal{Q} \mathcal{J}^{n} / \mathcal{N}(y) \mathcal{Q} \mathcal{J}^{n}\right)\right)
$$

is surjective, and consequently we have $H^{1}\left(U, \mathcal{N}(y) \mathcal{Q} J^{n}\right)=0$ for $y \in U_{x}$. Since $S$ is compact, there exists $n_{S}$ such that $H^{1}\left(U, \mathcal{N}(y) \mathcal{Q} J^{n}\right)=0$ for $y \in S$ and $n \geq n_{S}$.

Remark 2.7. Notice that for each $n \in \mathbb{N}$ the set

$$
A_{n}:=\left\{x \in X, \mathcal{Q J}_{x}^{n} \text { is not generated by } \Gamma\left(X, \mathcal{Q J}^{n}\right)\right\}
$$

is analytic and contained in $S$. The set

$$
A_{n, m}:=\bigcap\left\{\operatorname{supp}\left(\operatorname{Coker}\left(f_{1}, \ldots, f_{m}\right)\right), f_{1}, \ldots, f_{m} \in \Gamma\left(X, \mathcal{Q J}^{n}\right)\right\}
$$

is analytic. Since $A_{n}=\bigcap_{m \in \mathbb{N}} A_{n, m}$, the set $A_{n}$ is analytic also.

\section{Conic Stein neighbourhoods}

We are interested in the following situation: let $X$ be 1-convex space with an exceptional set $S$, let $Z$ be a complex space equipped with a metric $d$ compatible with the complex space topology, and let $h: Z \rightarrow X$ be a holomorphic submersion. Let $A \subset X$ be compact and holomorphically convex with $A \supset S$, possessing a basis of relatively compact 1-convex neighbourhoods $\left\{U_{l}\right\}$ in $X$ and $C \subset A$ a compact set, let $C \cap S=\emptyset$ and let $g: X \rightarrow \mathbb{C}$ be a holomorphic function with $g(S)=0$ and $g^{-1}(0) \cap C=\emptyset$.

Remark 3.1. In our application this situation occurs when gluing sections over Cartan pairs. The gluing takes place over the set $C$.

Let $a: X \rightarrow Z$ be a continuous section of the submersion, holomorphic on a neighbourhood $U$ of $A$. We want to find a Stein neighbourhood $V$ of $a\left(U \backslash g^{-1}(0)\right)$, such that the width of $V$ in the fibre direction decreases at most polynomially with the distance from $g^{-1}(0)$. We call this type of a neighbourhood a conic neighbourhood (along $g^{-1}(0)$ ). To obtain a conic neighbourhood we modify Demailly's theorem on neighbourhoods of $q$-complete spaces. 
Theorem 3.2 (Conic neighbourhoods). Let $a: X \rightarrow Z$ be a continuous section of the submersion, holomorphic on a neighbourhood $U$ of $A$ and let $g: X \rightarrow \mathbb{C}$ be a holomorphic function satisfying $g(S)=0$. Then for each relatively compact 1-convex subset $U^{\prime}$ of $U$ there exists a Stein neighbourhood of $a\left(U^{\prime} \backslash g^{-1}(0)\right)$ in $Z$ which is conic along $g^{-1}(0)$.

The idea of the construction is the following: since $U^{\prime}$ is 1-convex, it posesses a nonnegative plurisubharmonic exhaustion function $\varphi$, such that $\varphi^{-1}(0)=S, \varphi$ is strictly plurisubharmonic outside $S$ and such that $L \varphi$ is bounded from below by a positive constant near $\partial U$. We take the function $\varphi$ to be a pull back by the Remmert reduction $\pi$ of a strictly plurisubharmonic nonnegative exhaustion function $\varphi^{\prime}$ on $\pi\left(U^{\prime}\right)$ with zeroes only on $\pi(S)$ with prescribed minimal growth. Because $\pi$ is holomorphic, we have $L \varphi(v, v)=L \varphi^{\prime}(\pi(v), \pi(v))$, so the Levi form of $\varphi$ decreases polynomially with the distance from $S$. Note that the rate of degeneracy is determined by the geometry of $X$. The functions $\varphi$ and $g$ can be regarded as functions on $a\left(U^{\prime}\right)$ and $a(U)$, respectively. We extend them constantly along fibres to (holomorphic) functions on $Z_{U^{\prime}}$ and denote the extensions by the same letters, $\varphi$ and $g$. The function $g$ is holomorphic, and the function $\varphi$ is plurisubharmonic. It is strictly plurisubharmonic in the horizontal directions and degenerate in the vertical directions. We would like to correct it to a strictly plurisubharmonic exhaustion function on a suitable conic neighbourhood. This can be done in two steps. 1 . First, we add to the function $\varphi$ a function of type $f=\sum f_{i} \overline{f_{i}}$, which is defined on an open neighbourhood of $a(U)$, where $f_{i}-s$ are 'holomorphic to a high degree' in the fibre directions and zero on $h^{-1}(S) \cup a(U)$, in order to obtain a strictly plurisubharmonic function on a conic neighbourhood, which is 'an exhaustion in the horizontal direction'. 2. The function $\log f$ has logarithmic poles (only) along $a(U) \cup h^{-1}(a(S))$. In a suitably chosen conic neighbourhood of $a(U \backslash S)$ (conic along $a(S))$ the negative part of its Levi form decreases polynomially with the distance from $a(S)$, so for $M$ big enough, the function $G=\varphi+f-\log |g|^{2 M+2}+\log f$ is

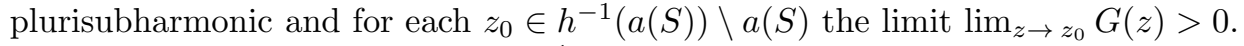

Now consider the sublevel set $G^{-1}([-\infty, c))$. For $c \ll 0$, the sublevel set is conic along $g^{-1}(0)$ and lies in the conic neighbourhood where the function is plurisubharmonic. Compose $G$ with a convex function $\chi$ with a pole in $c$ which is zero on $(-\infty, 2 c]$. The function $\chi \circ G$ is plurisubharmonic and 'exhausts the conic neighbourhood in the vertical direction'. The function $\varphi+f-\log |g|^{2 M+2}+\chi \circ G$ is then a strictly plurisubharmonic exhaustion function on a conic neighbourhood of $a\left(U^{\prime} \backslash g^{-1}(0)\right)$.

3.1. Construction of $f_{i}$ 's and their common zero set. If $U$ was Stein, there would be (finitely many) holomorphic functions $f_{i}$ on a Stein neighbourhood of $a(U)$ in $Z$, such that their zero set would be precisely $a(U)$. In general, this does not hold, but in the 1-convex case we can hope for 'functions, holomorphic to a high degree' defined on a conic neighbourhood of $a(U) \backslash h^{-1}(S)$ with zero set $a(U) \backslash h^{-1}(S)$, where $S$ is the exceptional set of $U$.

Consider the ideals $\mathcal{F}=\mathcal{J}^{m+1}(a(U))$ and $\mathcal{G}=\mathcal{J}(a(U))$ of $\mathcal{O}_{Z}$. Then the support of the sheaf $\mathcal{Q}=\mathcal{G} / \mathcal{F}=\mathcal{J}(a(U)) / \mathcal{J}^{m+1}(a(U))$ is $a(U)$, and the sheaf is also $\mathcal{O}_{a(U)^{-}}$ coherent. Since the sequence

$$
0 \rightarrow \mathcal{F} \rightarrow \mathcal{G} \rightarrow \mathcal{G} / \mathcal{F} \rightarrow 0
$$


is exact, each section $s \in \Gamma(a(U), \mathcal{G} / \mathcal{F})$ can be lifted locally to a section of $\mathcal{G}$, i.e. it can be represented locally as a holomorphic function on $Z$. By construction, such local lifts will coincide to the $m$-th order in the fibre directions (on $a(U)$ ).

The next problem is to find global sections that generate $\mathcal{Q}$ locally. In general, they do not. Such sections, however, exist if we allow multiple zeroes on the exceptional set. We may assume that $U$ is relatively compact.

By Theorem 2.5. there exist a $n_{0} \in \mathbb{N}$ such that for $n \geq n_{0}$ there are finitely many sections $\hat{f}_{i} \in \Gamma\left(a(U), \mathcal{Q J}^{n}\right)$ generating $\mathcal{Q J}^{n}$ at each point. Then for each $z \in a(U \backslash S)$ their local lifts $f_{i, z} \in \Gamma\left(V_{z}, \mathcal{G}\right)$ also generate $\mathcal{G}$ on some small open neighbourhood $V_{z} \subset Z$ of $z$. Fix such $n$.

To construct a conic neighbourhood of $a(U)$ we need the functions defined on a neighbourhood of $a(U)$. To obtain such functions we patch together their local holomorphic lifts. There exists a locally finite covering of $a(U)$ by product neighbourhoods $\left\{U_{j}\right\}$ such that each $\hat{f}_{i}$ has a holomorphic lift $f_{i j}$ on $U_{j}$.

Assume for a moment that $X$ and $S$ are manifolds. Let $(z, w)$ be local coordinates on $U_{j}, z=\left(z_{1}, z_{2}\right)$ in the $a(U)$ direction and $w$ in the fibre direction; moreover, let $a(S) \cap U_{j}=\left\{\left(z_{1}, z_{2}, 0\right), z_{2}=0\right\}$ (when the intersection is not empty). The monomials $w^{\alpha} z_{2}^{\beta},|\alpha|=1,|\beta|=k$ generate the $\mathcal{O}_{Z}$-coherent ideal $\mathcal{G J}\left(h^{-1}(S)\right)^{k}$ on a neighbourhood of $a(U)$; here $k=0$ if $\left|z_{2}\right| \neq 0$ and $k=n$ otherwise. Then the lifts can be chosen to be of the form $f_{i j}(z, w)=\sum_{|\alpha|=1,|\beta|=k} w^{\alpha} z_{2}^{\beta} a_{i \alpha \beta}\left(z_{1}, z_{2}, w\right)$, where $a_{i \alpha \beta}(z, w) \in P_{m-1}[\mathcal{O}(z)](w)$ (polynomials in $w$ of degree $m-1$ with coefficients in $\mathcal{O}(z))$. Let $\left\{\chi_{j}\right\}$ be a partition of unity subordinate to $\left\{U_{j} \cap a(U)\right\}$ and let

$$
f_{i}:=\sum \chi_{j} f_{i j}, \quad f=\sum\left|f_{i}\right|^{2} .
$$

By construction we have the estimate $f(z, w) \geq C_{j}|w|^{2}\left|z_{2}\right|^{2 k}$ on $U_{j}$ for some suitably chosen positive constant $C_{j}$ (depending on $U_{j}$ ). This also means that there exists an open neighbourhood $V \subset Z$ of $a(U)$, such that $f^{-1}(0) \cap V=a(U) \cup h^{-1}(a(S))$.

In the case of complex spaces we have the following local coordinates. Let $T X$ be a Zariski tangent space. Choose $x \in X$ and let $N=\operatorname{dim} T_{x} X$ be the embedding dimension of $X$ at $x$. Then there exists an open subset $U_{x}$ of $X$ and a proper holomorphic embedding $h_{x}: U_{x} \rightarrow B^{N}(0, \delta) \subset \mathbb{C}^{N}, h_{x}(x)=0$; our local coordinates will be the coordinates of $\mathbb{C}^{N}$.

Definition 3.3. Let $X, x, U_{x}, h_{x}$ and $N$ be as above. An upper semicontinuous function $f: X \rightarrow[-\infty, \infty), f \neq-\infty$, is (strictly) plurisubharmonic on $U_{x} \subset X$ if there exists an upper semicontinuous (strictly) plurisubharmonic function $\tilde{f}$ : $B^{N}(0, \delta) \rightarrow[-\infty, \infty)$, such that $\tilde{f} \circ h_{x}=f$.

Denote by $z$ the coordinates in the horizontal (e.g. $a(U)$ ) direction and by $w$ the coordinates in the vertical direction. The coordinates can be chosen such that $a(U) \cap U_{j}=\left\{(z, 0) \in U_{j}\right\}$. Let $\zeta=\left(\zeta_{1}, \ldots, \zeta_{\lambda}\right)$ be generators of the sheaf $\mathcal{J}(S)$ near $x$. Then the monomials $w^{\alpha} \zeta^{\beta},|\alpha|=1,|\beta|=k$ generate the $\mathcal{O}_{Z}$-coherent ideal $\mathcal{G J}\left(h^{-1}(S)\right)^{k}$ on a neighbourhood of $a(U)$. Here we can take $k=0$ if a neighbourhood of point from $a(U \backslash S)$ is considered (in local coordinates this means a point of the form $(z, 0)$ with $|\zeta|(z) \neq 0)$ and $k=n$ for neighbourhoods of points from $a(S)$.

3.2. The Levi forms of $f, \varphi+f$ and $\varphi+f+\log f$. Let $D$ denote the holomorphic derivative, $D f=\sum \frac{\partial f}{\partial s_{i}} d s_{i}$, and let $\bar{D}$ denote the antiholomorphic derivative, $\bar{D} f=$ 
$\sum \frac{\partial f}{\partial \bar{s}_{i}} d \bar{s}_{i}$. Then

$$
L f(v)=\sum\left|D f_{i}(v)\right|^{2}+\left|D \bar{f}_{i}(v)\right|^{2}+L f_{i}(v) \bar{f}_{i}+f_{i} L \bar{f}_{i}(v) .
$$

By construction the Levi form $L f(v)$ is strictly positive in the fibre directions on $a(U \backslash S)$, zero on $a(S)$, and its negative part is locally bounded and goes to 0 as we approach $a(U)$. We will show that $L f(v)$ is strictly positive in the fibre directions on a neighbourhood of $a(U \backslash S)$, which is conic along $a(S)$.

Let $(z, w)$ be local coordinates in $U_{j}$ such that $U_{j} \cap a(U)=\left\{(z, 0) \in U_{j}\right\}$ and $\zeta=\left(\zeta_{1}, \ldots, \zeta_{\lambda}\right)$ are the generators of the sheaf $\mathcal{J}(S)$. Then $a(S) \cap U_{j}=\{(z, 0) \in$ $\left.U_{j}, \zeta(z)=0\right\}$. Near a point from $a(S)$ the functions $f_{i}$ can be written in the form

$$
f_{i}(z, w)=\sum_{|\alpha|=1,|\beta|=n} w^{\alpha} \zeta^{\beta} c_{i \alpha \beta}(z, w)+\sum_{j,|\alpha|=m+1,|\beta|=n} w^{\alpha} \zeta^{\beta} c_{i j \alpha \beta}(z, w) \chi_{j}(z),
$$

where $c_{i \alpha \beta}$ and $c_{i j \alpha \beta}$ are holomorphic. As a result the following holds true:

$$
\begin{aligned}
\left|f_{i}\right| & =O\left(|w||\zeta|^{n}\right) \\
\left|D f_{i}\right| & =O\left(|\zeta|^{n}+|w||\zeta|^{n-1}\right), \\
\left|\bar{D} f_{i}\right| & =O\left(|w|^{m+1}|\zeta|^{n}\right), \\
\left|L f_{i}\right| & =O\left(|w|^{m}|\zeta|^{n}+|w|^{m+1}|\zeta|^{n-1}\right) .
\end{aligned}
$$

If we consider points in a conic neighbourhood along $S,|w| \leq c|\zeta|$ for some positive constant $c$, we obtain $\left|f_{i}\right|=O\left(|\zeta|^{n+1}\right),\left|D f_{i}\right|=O\left(|\zeta|^{n}\right),\left|\bar{D} f_{i}\right|=O\left(|\zeta|^{m+n+1}\right)$, $\left|L f_{i}\right|=O\left(|\zeta|^{m+n}\right)$.

The negative part of $L f$ is hidden in the terms $\sum L f_{i}(v) \bar{f}_{i}+f_{i} L \bar{f}_{i}(v)$ and is therefore bounded by $c_{1}|\zeta|^{m+1+2 n}$. Since the functions $f_{i}$ are locally generating the ideal $\mathcal{Q} J^{n}(a(S))$, the term $\sum\left|D f_{i}(v)\right|^{2}$ has the expansion

$\sum\left|D f_{i}(v)\right|^{2}=\sum_{|\beta|=n} c_{\beta}\left|\zeta^{\beta}\right|^{2}+\sum_{|\alpha|=1,|\beta|=n-1} c_{\alpha \beta}\left|w^{\alpha}\right|^{2}\left|\zeta^{\beta}\right|^{2}+$ terms of higher order,

where $c_{\beta}, c_{\alpha \beta}>0$, so it is bounded from below by $c_{2}|\zeta|^{2 n}$. The expansions and estimates for functions near points from $a(U \backslash S)$ are essentially the same. We just have to take the generator $\zeta=1$. Then we have $\left|f_{i}\right|=O(|w|),\left|D f_{i}\right|=O(1)$, $\left|\bar{D} f_{i}\right|=O\left(|w|^{m+1}\right),\left|L f_{i}\right|=O\left(|w|^{m}\right)$ and $\sum\left|D f_{i}(v)\right|^{2}>0$. Hence $f$ is strictly plurisubharmonic in the fibre directions on an open neighbourhood of $a(U \backslash S)$ which is conic along $a(S)$.

All the directions where the Levi form is negative have a nonzero projection to the horizontal direction, and on each relatively compact subset of $U$ the norms of projections of unit vectors in these directions are bounded from below by a strictly positive constant. In the horizontal directions we have $L \varphi=O\left(|\zeta|^{2 \mu}\right)$, so the function $\varphi+f$ is strictly plurisubharmonic on an open neighbourhood of $a\left(U^{\prime} \backslash S\right)$ which is conic along $a(S)$ and $L(\varphi+f)=O\left(|\zeta|^{2 \mu}\right)$ for $m$ large enough.

Now we claim that $\log f$ is almost plurisubharmonic on an open neighbourhood of $a(U \backslash S)$ which is conic along $a(S)$. 
Let $v \in T X$ be a unit tangential vector and

$$
\begin{aligned}
A & =\frac{1}{\sum f_{i} \bar{f}_{i}} \sum L f_{i}(v) \bar{f}_{i}+f_{i} L \bar{f}_{i}(v), \\
B & =+\frac{1}{\sum f_{i} \bar{f}_{i}} \sum\left|D \bar{f}_{i}(v)\right|^{2}, \\
C & =-\frac{1}{\left(\sum f_{i} \bar{f}_{i}\right)^{2}} \sum\left(\bar{f}_{i} \bar{f}_{j} \bar{D} f_{i}(v) D f_{j}(v)+\bar{f}_{i} f_{j} \bar{D} f_{i}(v) D \bar{f}_{j}(v)\right. \\
& \left.\quad+f_{i} f_{j} \overline{D f_{i}(v)} D \bar{f}_{j}(v)\right), \\
D & =+\frac{1}{2 \sum\left(f_{i} \bar{f}_{i}\right)^{2}} \sum\left|f_{j} D f_{i}(v)-f_{i} D f_{j}(v)\right|^{2} .
\end{aligned}
$$

Then $L \log f(v)=A+B+C+D$. For $f$ we have the estimate $f \geq c_{3}|w|^{2}|\zeta|^{2 n}, c_{3}>0$. The terms $B$ and $D$ are nonnegative. Using estimates above we obtain

$$
A=O\left(|w|^{m-1}\right), \quad B=O\left(|w|^{2 m}\right), \quad C=O\left(|w|^{m-1}\right) ;
$$

the term $D$ is zero in some directions and grows as $|w|^{-2}$ in some other. For $m \geq \mu$ the negative part of the Levi form goes to 0 when approaching $a(U)$. Therefore, the function $\varphi+f+\log f$ is strictly plurisubharmonic on a neighbourhood of $a\left(U^{\prime} \backslash S\right)$. We claim that the neighbourhood can be chosen conic along $a(S)$. Because we have assumed that $|w| \leq c_{1}|\zeta|$, the negative part of $L \log f$ is $O\left(|\zeta|^{m-1}\right)$. The same estimate is obtained on neighbourhoods of points from $a(U \backslash S)$. Since $L(\varphi+f)=$ $O\left(|\zeta|^{2 \mu}\right)$ the sum $\varphi+f+\log f$ is strictly plurisubharmonic on a conic neighbourhood of $a\left(U^{\prime} \backslash S\right)$ along $a(S)$, provided that $m \geq 2 \mu+2$.

3.3. Proof of the theorem on existence of conic neighbourhoods. With all the ingredients at our disposal we can now complete the proof of the Theorem 3.2. The sublevel sets of the function $\varphi+f+\log f$ contain the fibres above $S$. To correct that, we choose a function $g \in \mathcal{O}(a(U))$, such that $N(g) \supset a(S)$, and $g^{-1}(0) \cap a(C)=\emptyset$. Consider the function

$$
G=\varphi+f+\log f-\log |g|^{2 l}
$$

for some $l \geq n+1$. It is easy to see that for $z_{0} \in h^{-1}(S) \backslash a(S)$ the limit $\lim _{z \rightarrow z_{0}} G(z)=\infty$ (the order of zeroes of $|g|^{2 l}$ is larger than the order of zeroes of $f$ ). Since $\log |g|^{2 l}$ is pluriharmonic, the function $G$ remains strictly plurisubharmonic in a conic neighbourhood. For the sublevel set $G^{-1}([-\infty, c))$ we get

$$
f \leq e^{c-\varphi-f}|g|^{2 l} .
$$

Near a point from $a(S)$ the following holds:

$$
|w|^{2} \leq \alpha e^{c-\varphi(z)-f(z, w)}|\zeta|^{-2 n}|g|^{2 l} .
$$

This means that such sublevel sets are conic along $a(S)$ (lie between two polynomial cones). On the complement of the set $a(S)$ the neighbourhood is of the form $|w| \leq \beta|g|^{2 l}$. For $l$ large enough (and $c$ small enough) the sublevelset also lies in the conic neighbourhood where $G$ is plurisubharmonic. Compose $G$ with a convex function $\chi$ with a pole in $c$ which is zero on $(-\infty, 2 c]$. The function $\chi \circ G$ is plurisubharmonic and exhausts the conic neighbourhood in the vertical direction. The function $\varphi+f-\log |g|^{2 l}+\chi \circ G$ is then a strictly plurisubharmonic exhaustion function on a conic neighbourhood of $a\left(U^{\prime} \backslash g^{-1}(0)\right)$. 


\section{Proof of the MAIN TheOREM}

4.1. Scheme of the proof of Theorem 1.1 in the case where $X$ is a manifold and $h: Z \rightarrow X$ is a fibre bundle. If $X$ is a manifold and $h: Z \rightarrow X$ a holomorphic fibre bundle, the scheme of the proof is significantly simpler than in the general case, and it is explained separately for the sake of clarity. The scheme follows the one described in FP1].

(1) Choose a locally finite open covering $\left\{U_{i}\right\}$ of the space $X$ and holomorphic functions $g_{1}, \ldots, g_{m}: X \rightarrow \mathbb{C}$ such that

$(\alpha) U_{0} \supset K, \overline{U_{i}} \cap K=\emptyset$ for $i \geq 1$,

( $\beta) a_{0}$ is holomorphic on $U_{0}$,

$(\gamma)$ the Runge condition holds for sections $U_{i} \rightarrow Z, i \geq 1$,

$(\delta) Z$ is trivial over $U_{i}, i \geq 1$,

$(\varepsilon)$ the common zero set of the functions $g_{1}, \ldots, g_{m}$ is $S$, and for $i \geq 1$ there exists $j \in\{1, \ldots, m\}$ such that $\bar{U}_{i} \cap g_{j}^{-1}(0)=\emptyset$.

(2) Using the Remmert reduction choose a plurisubharmonic exhaustion function $\rho: X \rightarrow \mathbb{R}$ such that $\rho^{-1}(0)=K$ and $\rho$ is strictly plurisubharmonic outside $K$.

(3) Construct a sequence of strongly pseudoconvex compact sets $A_{i}, K \subset \operatorname{int} A_{0}$, such that $A_{i+1}=A_{i} \cup B_{i}$, where $B_{i} \subset U_{j}$ is either a pseudoconvex bump on $A_{i}$ or a thin handle attached to $A_{i}$ (Lemma 12.3 in [HL, Theorem 3.1 in [F3]). These sets are sublevel sets of appropriate plurisubharmonic functions, which are strictly plurisubharmonic outside $K$.

(4) Enlarge the region where the continuous section is holomorphic, inductively. Assume that $a_{0}$ is holomorphic on a neighbourhood $A=A_{i}$ and continuous elsewhere. Construct a homotopy $a_{t}$ of continuous sections such that each $a_{t}$ approximates $a_{0}$ on $A$ and such that $a_{1}$ is holomorphic on a neighbourhood of $A \cup B=A_{i+1}$.

(a) There exists a local spray on $A$ which dominates on $A \backslash S$ and keeps $S$ fixed. In other words, there exists a fibre-preserving holomorphic map $F: U_{A} \times B_{n}(0, \delta) \rightarrow Z, U_{A}$ being an open neighbourhood of $A$, such that $F(x, \cdot): B_{n}(0, \delta) \rightarrow Z_{x}, F_{t}(x, t)$ is surjective for $t=0$ and $x \in A \backslash S$, $F(x, 0)=a(x)$ and $F(x, t)=F(x, 0)$ for all $x \in S$.

(b) There exists a fibre-preserving map $G: U_{B} \times B_{n}(0, \delta) \rightarrow Z$ such that $G$ approximates $F$ on $C=A \cap B ; U_{B}$ is an open neighbourhood of $B$. If $B$ is a bump, then the approximation is possible because of the Runge condition on the submersion. Recall that if $B$ is a bump, then $C$ is holomorphically contractible in $B$ and $\left.Z\right|_{U_{B}}$ is trivial. This provides the holomorphic homotopies needed for the application of the Runge condition. If $B$ is a thin handle, the existence of approximation is given by Theorem 3.2 in ([F3]). The theorem provides an approximation over a thin handle $B^{\prime}$ attached to $C$. In principle it might be thinner than $B$. If this is the case, we can get from $B^{\prime}$ to a neighbourhood of $B$ using bumps which give a map $G: U_{B} \times B_{n}(0, \delta) \rightarrow Z$ approximating $F$ on $C \times B_{b}\left(0, \delta^{\prime}\right)$.

(c) There exists a map $\gamma=\left(i d_{x}, c\right): U_{C} \times B_{n}\left(0, \delta^{\prime}\right) \rightarrow U_{C} \times B_{n}\left(0, \delta^{\prime}\right)$ close to identity such that $F=G \circ \gamma$ on $U_{C} \times B_{n}\left(0, \delta^{\prime}\right)$. Near the zero section the map $\gamma$ has a decomposition $\gamma=\beta \circ \alpha^{-1}$, where $\alpha: U_{A} \times B_{n}\left(0, \delta^{\prime}\right) \rightarrow$ $U_{A} \times B_{n}\left(0, \delta^{\prime}\right)$, and $\beta: U_{B} \times B_{n}\left(0, \delta^{\prime}\right) \rightarrow U_{B} \times B_{n}\left(0, \delta^{\prime}\right)$ are invertible on a neighbourhood of the zero section over $U_{C}$. 
(d) We have $F \circ \alpha(x, 0)=G \circ \beta(x, 0)$ for $x \in U_{C}$. This defines a holomorphic section $a_{1}$ over $A \cup B$, homotopic to $a_{0}$ on a neighbourhood of $A \cup B$. Outside $A \cup B$ the section $a_{1}$ can be glued to the section $a_{0}$ by a homotopy, thus yielding a continuous section $a_{1}$, holomorphic on a neighbourhood of $A \cup B$, homotopic to $a_{0}$ and approximating $a_{0}$ on $A$. Since $F(x, t)=F(x, 0)$ for all $x \in S$ we also have $a_{t}(x)=a_{0}(x)$ on $S$ ( $a_{t}$ is the homotopy).

(5) In the limit we get the desired holomorphic section.

4.2. Details. (4a) By the definition of the initial open covering there exists $g \in$ $\left\{g_{1}, \ldots, g_{m}\right\}$ such that $g^{-1}(0) \cap U_{B}=\emptyset$. Let $U_{A}$ be a 1-convex neighbourhood of $A$ and $V$ a conic Stein neighbourhood of $a\left(U_{A} \backslash g^{-1}(0)\right)$. By Theorem A for 1-convex spaces (Theorem 2.5) for each $k \in \mathbb{N}$ large enough there exist finitely many vector fields $h_{1}, \ldots, h_{n}$ of the bundle $\left.V T(Z)\right|_{a\left(U_{A}\right)}$ with zeroes of order (at least) $k$ on $a\left(g^{-1}(0)\right)$ generating $\left.V T(Z)\right|_{a\left(U_{A} \backslash g^{-1}(0)\right)}$. We extend these vector fields on $V$ and integrate them. Since $V$ is conic, the fields can be integrated for sufficiently small times $t \leq t_{0}$ for all $x \in A \backslash S$ (provided $k$ is large enough). Because of the zeroes on $a\left(g^{-1}(0)\right)$, we can extend the flows of the fields on $a\left(g^{-1}(0)\right)$, thus obtaining a map $F: U_{A} \times B_{n}(0, \delta) \rightarrow Z$ fulfilling all the requirements. Note that this construction also works for a complex space.

(4c) Since $U_{C}$ is Stein, we have a decomposition $U_{C} \times \mathbb{C}^{n}=\operatorname{ker} D F \oplus N$. For $t \in \operatorname{ker} F$ sufficiently small, the maps $F, G: U_{C} \times\left((N \oplus t) \cap B_{n}(0, \delta)\right) \rightarrow Z$ are injective. Moreover, if $G$ is sufficiently close to $F$ we have a uniquely defined map $\gamma_{t}: U_{C} \times\left((N \oplus t) \cap B_{n}\left(0, \delta_{1}\right)\right) \rightarrow U_{C} \times\left((N \oplus t) \cap B_{n}\left(0, \delta_{1}\right)\right)$ which is close to the identity for $\delta_{1}$ sufficiently small. Obviously, the maps $\gamma_{t}$ are holomorphic in all variables, and define the map $\gamma: U_{C} \times B_{n}\left(0, \delta^{\prime}\right) \rightarrow U_{C} \times B_{n}\left(0, \delta^{\prime}\right)$ for sufficiently small $\delta^{\prime}$. If $F$ and $G$ coincide, then we have $\gamma=i d$. The desired decomposition of $\gamma$ is obtained from an implicit function theorem (Proposition 5.2 in [FP1]). In this theorem a solution of a $\bar{\partial}$ equation with uniform estimates on a neighbourhood of a strictly pseudoconvex set $A \cup B$ with support disjoint from $A \backslash B$ is used where $A \cup B$ is a subset of a Stein manifold. In the 1-convex case the same can be done with uniform estimates using the Remmert reduction, since the supports of the forms we deal with do not intersect $S$. In addition, we can require that the solutions of the $\bar{\partial}$ equation are zero on $S$ without spoiling the uniform estimates.

4.3. Proof of Theorem 1.3 if $X$ is a complex space and $h: Z \rightarrow X$ is a submersion. In this case we cannot directly use the Henkin-Leiterer bump method (Cartan pairs). The notion of Cartan pairs can (and will be) generalized to the case of a complex space, but even with this generalization we will still be in trouble when trying to use the Runge property. Let us recall that in section 4.1 $(4)(b)$ it was the structure of the manifold that provided the homotopies necessary for the application of the Runge property. Here we have to start in such a way that we have all the homotopies needed at our disposal from the very beginning. This is the reason why we have to use holomorphic complexes, prisms and Cartan strings as in ([FP2] ) and have to follow the procedure described there. The aim of this section is not to present the complete proof of the Theorem 1.3 but merely to explain those parts of the proof which differ from the proof in FP2] in the 1-convex case. 
Let us recall the initial data: $X^{\prime} \subset X$ is an analytic set, $S \subset X$ the exceptional set, the parameter space $P$ a Hausdorff compact set, $P_{0} \subset P_{1} \subset P, P_{0}$ a closed set, $P_{1}$ an open set and $P_{0}$ a nice subset of $P, g_{1}, \ldots, g_{m}: X \rightarrow \mathbb{C}$ holomorphic functions with $S$ as a common zero set, $G=\bigcup g_{i}^{-1}(0), a_{0, p}: X \rightarrow Z, p \in P$ a given family of continuous sections, holomorphic on a neighbourhood of a holomorphically convex compact set $K \supset S$ and such that $a_{0, p}(x)=a_{0, q}(x)$ for each $p, q \in P, x \in G$, $\left.a_{0, p}\right|_{X^{\prime}}$ is holomorphic and $a_{0, p}$ is holomorphic for $p \in P_{1}$. We will also assume that $X^{\prime} \supset G$.

The scheme: 1. Construct the initial 1-prism using Cartan strings. 2. Construct a continuous family of local sprays. 3. Enlarge the region where the initial sections are holomorphic, inductively (compare $4.1(4)$ ). 4. Modify the prisms in order to obtain a continuous family of holomorphic sections.

(1) To begin with, choose a locally finite covering $\left\{U_{i}\right\}$ of $X$ as in 4.1 (1), skipping the condition $(\delta)$. For every relatively compact Stein open set $L \subset \pi(X)$ there exists an embedding of $L$ into a Euclidean space. Using this fact we can for every open covering with the above mentioned conditions construct an increasing sequence of holomorphically convex subsets $A_{0}, A_{1}, A_{2}, \ldots, \cup A_{i}=X$ such that

(1) $K \subset \operatorname{int} A_{0}$.

(2) For every $n \in \mathbb{N}$ there exists an embedding $\iota_{n}$ of a neighbourhood of $\pi\left(A_{0} \cup \ldots \cup A_{n}\right)$ into a complex manifold and a Cartan string $\hat{A}_{0}, \ldots, \hat{A_{n}}$ in this manifold such that $\iota_{n}\left(\pi\left(A_{i}\right)\right)=\hat{A}_{i} \cap \iota_{n}\left(\pi\left(A_{0} \cup \ldots \cup A_{n}\right)\right.$,

The sequence $A_{0}, \ldots, A_{n}$ will also be called a Cartan string of length $n+1$. If $X$ is a manifold, then this definition of a Cartan string coincides with the initial one.

Since we are dealing with a submersion $Z \rightarrow X$, we can construct the initial holomorphic complex similarly as in FP2, Proposition 4.7. The only thing needed there is a local product structure of the submersion, and this is independent of the fact that in this case the base space was a manifold. It should be mentioned though that the family of the initial complexes must be continuous with respect to parameter $p$ and that the small holomorphic sections must coincide with the initial ones on $X^{\prime}$. To achieve this we have to impose some additional conditions on the initial open covering. For $x \in X \backslash K$ choose an open neighbourhood $U_{x} \subset X$ of $x \in X$ such that there exist finitely many open sets $V_{i} \subset Z$ and holomorphic maps $h_{i}: U_{x} \times B^{n}(0,1) \rightarrow Z, i=1, \ldots, m$, and satisfying $\bigcup_{p \in P} a_{0, p}\left(U_{x}\right) \subset \cup V_{i}$. Then construct an open covering $\left\{\tilde{Q}_{i}\right\}$ of $P$ satisfying $\tilde{Q}_{0}=P_{1}, \tilde{Q}_{i}=\{p \in P \backslash$ $\left.P_{0}, a_{0, p}\left(U_{x}\right) \subset V_{i}\right\}$. Let $\left\{Q_{i}\right\}$ be a covering with closed sets such that $Q_{i} \subset \tilde{Q}_{i}$ and $P_{0} \subset \operatorname{int} Q_{0}$. Let $\left\{\chi_{i}\right\}$ be a partition of unity on $P$ such that supp $\chi_{0}=Q_{0}$ and $\operatorname{supp} \chi_{i} \subset Q_{i}$. Let $b_{i, p}(y):=h_{i}(y, 0), y \in U_{x}$, if $p \in Q_{i}, i \geq 1$, and $b_{i, p}=a_{i, p}$ for $p \in Q_{0}$. Let $i(p):=\min \left\{i, p \in Q_{i}\right\}$. In the local chart $h_{i(p)}$ define

$$
\tilde{a}_{1, p}:=\sum_{i} \chi_{i}(p) b_{i, p}
$$

The result is a continuous family of holomorphic sections. To keep the sections fixed on $X^{\prime}$, we have to correct the sections using a bounded linear extension operator given by Lemma 3.1 in [FP3]. First, we embed $U_{x}$ into $C^{N}$. Denote the embedding by $\iota$ and let $\iota(x)=0$. There exists a bounded linear extension operator $\tilde{E}: \mathcal{O}\left(\iota\left(X^{\prime}\right)\right) \rightarrow \mathcal{O}\left(B^{N}(0,1)\right)$ that (after shrinking $\left.U_{x}\right)$ gives rise to a bounded 
linear extension operator $E: \mathcal{O}^{n}\left(X^{\prime}\right) \rightarrow \mathcal{O}^{n}\left(U_{x}\right)$. In the local chart $h_{i(p)}$ define

$$
a_{1, p}:=\sum_{i} \chi_{i}(p) E\left(\left.\left(a_{0, p}-a_{1, p}\right)\right|_{X} ^{\prime}\right)+a_{1, p}
$$

(2) Construction of a continuous (smooth) family of local sprays.

Lemma 4.1 (A continuous (smooth) family of local sprays). Let $K \subset X$ be a holomorphically convex compact set containing $S, U \subset \bar{U} \subset U^{\prime}$ its open neighborhoods in $X$ and $g: X \rightarrow \mathbb{C}$ a holomorphic function, which is zero on $S$. Let $a_{p}: U \rightarrow Z$ be a continuous (smooth) family of holomorphic sections of $Z$ satisfying $a_{p}(x)=a_{q}(x)$ for $p, q \in P, x \in G$. Then there exist a continuous (smooth) family of vector fields $v_{1, p}, \ldots, v_{k, p}$ of $V T(Z)$ over a conic neighbourhood $U_{p}$ of $a_{p}\left(U \backslash g^{-1}(0)\right)$ and a $t_{0}>0$ such that they generate $\left.V T(Z)\right|_{U_{p}}$, and their flows stay in $U_{p}$ for times $|t|<t_{0}$ and points from a smaller conic neighbourhood $U_{p}^{\prime} \subset U_{p}$.

Proof. The lack of compactness of the set $\bigcup_{p} a_{p}\left(U \backslash g^{-1}(0)\right)$ is compensated by the fact that $a_{p}(x)=a_{q}(x)$ for $p, q \in P, x \in G$. Then every covering of $\bigcup_{p} a_{p}\left(U \backslash g^{-1}(0)\right)$ by conic neighbourhoods has a finite subcovering. The rest of the proof is the same as in Proposition 4.3 of FP1.

(3) The only thing that remains to be proved is the gluing lemma for complex spaces. Let $(A, B)$ be a Cartan pair in a complex space, $A \subset U$ and $B \subset V, U, V \subset$ $X$ open and $V$ Stein and assume that we already have constructed a continuous family of holomorphic sections $a_{p}: U \rightarrow Z, p \in P$. By the Runge property it can be approximated on $A \cap B$ arbitrarily well by a continuous family of holomorphic sections $b_{p}: V \rightarrow Z$ such that $b_{p}(x)=a_{0, p}(x)$ for $x \in X^{\prime} \cap V$ and $b_{p}=\left.a_{0, p}\right|_{V}$ for $p \in P_{1}$. There also exists a continuous (smooth) family of local sprays $s_{p}$ : $U \times B^{N}(0,1) \rightarrow Z$ with $s_{p}(\cdot, 0)=a_{p}$ and a continuous family of sprays $t_{p}:$ $V \times \mathbb{C}^{n} \rightarrow Z$ with $t_{p}(\cdot, 0)=b_{p}$. As in Lemma 5.4 of [FP1 this gives rise to a family of gluing maps $\psi_{p}:(U \cap V) \times B^{N}(0,1) \rightarrow \mathbb{C}^{N}$ satisfying $s_{p}(x, u)=t_{p}\left(x, \psi_{p}(x, u)\right)$, $\psi_{p}\left(x^{\prime}, 0\right)=0$ for $x^{\prime} \in X^{\prime}$ and $\psi_{p}(x, u)=u$ for $p \in P_{1}$. With some additional work (see the proof of Proposition 5.2 of [FP1]) we may assume that the gluing maps are close to $(x, u) \rightarrow u$ for $x \in C$. The next step is the splitting. To do the splitting (Proposition 4.1 in FP3]) the base space has to be a manifold because of solving the $\bar{\partial}$-equation. We now transfer our gluing maps to Euclidean space. By the definition of a Cartan pair there exists a Cartan pair $(\hat{A}, \hat{B})$ in some $\mathbb{C}^{m}$ and an embedding $\iota$ of an open neighbourhood $U$ of $\pi(A \cup B)$ into $\mathbb{C}^{n}$ such that $\iota(\pi(A))=\hat{A} \cap \iota U$ and $\iota(\pi(B))=\hat{B} \cap \iota U$. Using the bounded linear extension operator constructed in Lemma 3.1 of [FP3, we extend the maps $\psi_{p}$ (which are close to $(x, u) \rightarrow u$ ) to some small neighbourhood of $\hat{A} \cap \hat{B}$ such that the extensions still are sufficiently close to $(x, u) \rightarrow u$. Moreover, by shrinking $P_{1}$ the extensions for $p \in P_{1}$ may be assumed to be $(x, u) \rightarrow u$. We now apply Proposition 4.1 in FP3 to get the continuous family of splittings. The operators $\mathcal{A}^{\prime}$ and $\mathcal{B}^{\prime}$ yielded by this proposition satisfy the following. If we denote $\alpha_{p}:=\mathcal{A}^{\prime}\left(\psi_{p}\right): U \rightarrow \mathbb{C}^{N}$ and $\beta_{p}:=\mathcal{B}^{\prime}\left(\psi_{p}\right): V \rightarrow \mathbb{C}^{N}$, then $\left.\alpha_{p}\right|_{U \cap X^{\prime}}=0$ and $\left.\beta_{p}\right|_{V \cap X^{\prime}}=0$. Define $a_{1, p}(x):=s_{p}\left(x, \alpha_{p}(x)\right)$ for $x \in U$ and $a_{1, p}(x)=t_{p}\left(x, \beta_{p}(x)\right)$ for $x \in V$. Because we have $\psi_{p}(x, \alpha(x))=\beta(x)$, both definitions coincide on $U \cap V$ and define a global section of $\left.Z\right|_{U \cup V}$. Consequently we obtain a continuous family of holomorphic sections of $h: Z \rightarrow X$ defined over a 
neighbourhood of $A \cup B$ and approximating the sections $a_{p}$ on $A$ and keeping the sections fixed for $x^{\prime} \in X^{\prime}$ or $p \in P_{1}$.

(4) Modifying the prisms to get homotopies of sections is now a purely combinatorial problem and does not have anything to do with the structure of the base space (see section 5 in [FP2]).

\section{ACKNOWLEDGEMENTS}

The author would like to thank Professor Franc Forstnerič for discussions that provided the stimulus for this paper.

\section{REFERENCES}

[AG] Andreotti, A., Grauert, H., Theoremes de finitude pour la cohomologie des espaces complexes. Bull. de la S.M.F., 90, p. 193 - 259 (1962). MR0150342 (27:343)

[CM] Camacho, C., Movasati, H., Neighborhoods of analytic varieties in complex manifolds. Monographs of the Institute of Mathematics and Related Sciences, 35, Lima, Peru, 2003. MR2010707 (2004j:32008)

[Ca] Cartan, H., Quotients of complex analytic spaces. International Colloquium on Function Theory, Tata Institute p. 1-15 (1960). MR0139769 (25:3199)

[Co] Colţoiu, M., On the Oka-Grauert principle for 1-convex manifolds. Math. Ann. 310, p. 561569 (1998). MR.1612254 (99c:32040)

[De] Demailly, J.-P., Cohomology of $q$-convex spaces in top degrees. Math. Z. 204, p. 283-295, (1990). MR.1055992 (91e:32014)

[F1] Forstnerič, F., Runge approximation on convex sets implies the Oka property. Ann. of Math. 163 (2006). MR2199229 (2006j:32011)

[F2] Forstnerič, F., The homotopy principle in complex analysis: A survey. Contemporary Mathematics 332, AMS (2003). MR2016091 (2004m:32047)

[F3] Forstnerič, F., Holomorphic submersions from Stein manifolds Annales Inst. Fourier, 54 (2004). MR2134229 (2005m:32019)

[FP1] Forstnerič, F., Prezelj, J., Oka's principle for holomorphic fiber bundles with sprays. Math. Ann. 317, p. 117 - 154 (2000). MR1760671 (2001m:32040)

[FP2] Forstnerič, F., Prezelj, J., Oka's principle for holomorphic submersions with sprays. Math. Ann. 322, 633-666 (2002). MR 1905108 (2003d:32027)

[FP3] Forstnerič, F., Prezelj, J., Extending holomorphic sections from complex subvarieties. Math. Z. 236, p. 43-68 (2001). MR1812449 (2002b:32017)

[FS] Forstnerič, F., Slapar, M., Stein structures and holomorphic mappings. Math. Z., 256, p. 615-646 (2007). MR2299574 (2008g:32036)

[G1] Grauert, H., Über Modifikationen und exzeptionelle analytische Mengen. Math. Ann. 146, p. 331-386 (1962). MR0137127 (25:583)

[G2] Grauert, H., The coherence of direct images. L'Enseignement math. 14, p. 99-119 (1968). MR0236422 (38:4718)

[GR1] Grauert, H. in Remmert, R., Theory of Stein Spaces. Grundl. Math. Wiss. 227, SpringerVerlag (1979). MR.580152 (82d:32001)

[GR2] Grauert, H. in Remmert, R.: Coherent analytic sheaves. Grundl. Math. Wiss. 265, SpringerVerlag (1984). MR0755331 (86a:32001)

[Gro] Gromov, M., Oka's principle for holomorphic sections of elliptic bundles. J. of the AMS 2, p. 851-897 (1989). MR1001851 (90g:32017)

[HL] Henkin, G.M., Leiterer, J., The Oka-Grauert principle without induction over the base dimension. Math. Ann. 311, p. 71-93 (1998). MR.1624267 (99f:32048)

[LV] Leiterer, J., Vâjâitu, V., A relative Oka-Grauert principle on 1-convex spaces. J. Reine Angew. Math. 564, p. 85-104 (2003). MR2021035 (2004j:32015)

[S1] Siu, Y.-T., The 1-convex generalization of Grauert direct image theorem. Math. Ann., 38, p. 203-214 (1971). MR0279341 (43:5063) 
[S2] Siu, Y.-T., Every Stein subvariety admits a Stein neighbourhood. Invent. Math., 38, p. 89100 (1976). MR0435447 (55:8407)

[Va] Vâjâitu, V., A characterization of 1-convex spaces. J. Math. Pures Appl. 84, p. 189-197 (2005). MR2118838 (2006i:32013)

Faculty of Mathematics and Physics, University of Ljubljana, Jadranska 19, SI-1000 LuUbluana, Slovenia

E-mail address: jasna.prezelj@fmf.uni-lj.si 